\title{
A GRAVITATIONALLY INDUCED (MACHIAN) MAGNETIC FIELD
}

\author{
J. EHLERS \\ University of Texas at Austin. USA \\ and \\ W. RINDLER \\ University of Texas at Dallas, USA
}

Received 11 June 1970

\begin{abstract}
The magnetic field induced by a rotating mass-shell having at its center a stationary charged sphere is calculated in the framework of linearized general relativity.
\end{abstract}

A well-known consequence of Einstein's theory of gravitation is the dragging of inertial frames by rotating massive bodies. This was first established by Thirring [1] in 1918. More recently that topic has received further attention from Bass and Pirani [2], Hönl and Maue [3], Brill and Cohen [4], and others. It is to be noted that the dragging effect is not necessarily always small: if pulsars are rotating neutron stars, as suspected, then inertial frames at their surface are dragged around several times per sec [5].

Thirring's particular investigation concerned the gravitational field inside a massive rotating shell in an otherwise empty and asymptotically flat universe. Part of the motivation stemmed from what Einstein had termed 'Mach's principle', according to which a gravitational theory should be so constructed that the same consequences result from assuming. for example. that a certain system is rotating within a fixed universe, or that the universe is rotating around that same system at rest. Thus the rotation of the universe relative to the earth would gravitationally cause the Coriolis and centrifugal fields experienced by observers on earth. By extension. one might expect the field inside a large rotating mass-shell to have Coriolis and centrifugal features. And this, indeed, was Thirring's finding.

Analogously, one of us conjectured [6] that an electrically charged stationary shell inside the rotating shell would be surrounded by a magnetic field. This conjecture poses a well-definable problem in general relativity. which we have solved in the linear approximation. It might be called the 'electromagnetic Thirring problem': given an asymptotically flat space-time whose only content is a charged spherical shell surrounded by a neutral massive shell. the first at rest and the second uniformly rotating relative to infinity, to find the resulting magnetic field.

A similar problem but with a central pointcharge in place of our spherically extended charge has been solved by Hofmann [7] (correctly) and by Dehnen [8] (incorrectly). It would appear to have no direct Machian significance.

Our method was the standard perturbation procedure. Let $G=$ gravitational constant. $Q=$ electric charge on the inner shell, $a=$ its radius. $M=$ mass of the outer shell. $R=$ its radius. $\omega=$ its angular velocity. We imagine a family of solutions of the coupled Einstein-Maxwell field equations. depending analytically on the parameters $G$ and $\omega$, for fixed values of $Q, M, a, R$. We compute the equations governing the gravitational field and the sources, to first order in $G$ and second order in $\omega$. We follow Hönl and Maue [3] in assuming the outer shell to be stressed only tangentially along the parallels of latitude, and to have spherical shape and uniform proper density when in motion. The per turbation of the gravitational field (metric) then turns out to be the superposition of the Thirring field (as corrected by Hönl and Maue for the stress in the shell) and the linearized ReissnerNordström metric [9]. suitably continued into the interior of the charged shell. The latter is assumed to carry Poincaré stress to balance its Coulomb repulsion, but no 'material' mass. The zero-order electromagnetic field is the ordinary Coulomb field of the static charged sphere. The perturbed electromagnetic field is found to con- 


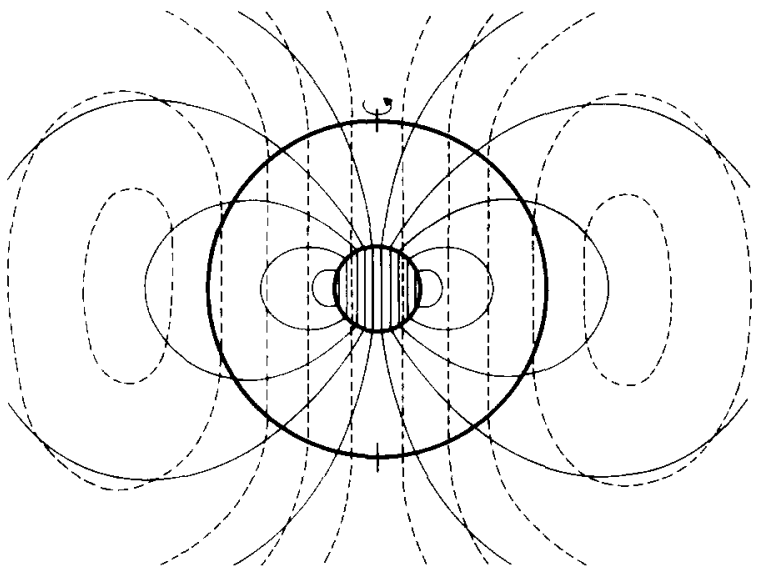

Fig. 1. Field lines of the components $\boldsymbol{B}$ (full) and $\boldsymbol{B}$. (stippled) of the magnetic field induced by a rotating mass-shell on the coulomb field of an enclosed concentric stationary charged shell.

sist of an electrostatic part of lesser immediate interest, and a magnetic part which may conveniently be divided into two components $B$ and $\boldsymbol{B}^{\prime}$ corresponding to two distinct source terms. Writing $r$ for the position vector from the common center of the two shells, and using Gaussian units and dimensions, we obtain

$$
\text { c } \boldsymbol{B}= \begin{cases}\frac{2}{3} Q a^{-1} \omega^{\prime} . & (r \cdot a), \\ Q a^{2}\left[\left(\omega^{\prime} \cdot r\right) r^{-5} r-\frac{1}{3} r^{-3} \omega^{\prime}\right] . & (r \cdot a),\end{cases}
$$

where $\omega^{\prime}=4 G M \omega / 3 R c^{2}$; this $\omega^{\prime}$ is the

'Thirring' angular velocity, which has the following significance: in a frame rotating at angular velocity $\omega^{\prime}$ the Coriolis field vanishes everywhere inside the massive shell. (In fact. in the Thirring problem the metric is flat inside that shell to first order in $\omega$. and $\omega^{\prime}$ is the angular velocity of the central inertial frame relative to infinity.) For the other component, $\boldsymbol{B}^{\prime}$, we find

$$
c \boldsymbol{B}^{\prime}=\left\{\begin{array}{lr}
\frac{1}{2} Q R^{-1} \omega^{\prime}, & (r \cdot R), \\
3 Q R^{2}\left[\left(\omega^{\prime} \cdot \boldsymbol{r}\right)\left(r^{-5}-R r^{-6}\right) \boldsymbol{r}-\frac{1}{3}\left(r^{-3}-\frac{3}{2} R r^{-4}\right) \omega^{\prime}\right] .
\end{array}\right.
$$$$
(r>R) \text {. }
$$

The field $\boldsymbol{B}$ is precisely the magnetic field of a uniformly charged shell of total charge $Q$ and radius $a$ rotating at angular velocity $\omega^{\prime}$ : outside the shell it is a dipole field and inside it is homogeneous. This, of course, is just the field expected on Machian grounds, given the Thirring effect. The field $\boldsymbol{B}^{\prime}$ is physically less obvious. Its sources are circular currents whose axes coincide with $\omega$, and which fill the exterior of the rotating shell. $B^{\prime}$ is homogeneous inside that shell, and outside of it the leading terms (those falling off like $r^{-3}$ ) constitute a dipole field of moment $Q R^{2} \omega^{\prime} / c$. (For $r^{2} \cdots 3 R^{2}$ this field is identical with that of a charged shell of radius $\sqrt{3} R$ and total charge $Q$ rotating with the Thirring velocity $\omega^{\circ}$.) Inside and near the charged shell $\boldsymbol{B}$ dominates $\boldsymbol{B}^{\prime}$ by a factor $\sim R / a$. The field $\boldsymbol{B}$ ' was already obtained by Hofmann $[7]$. Since $\boldsymbol{B}$ vanishes in the limit $a \cdot 0, \boldsymbol{B}^{\prime}$ is the correct solution for a point-charge.

Unfortunately the effect discussed here seems to be many orders of magnitude too small to be measured in the laboratory.

Details and other consequences will be published elsewhere.

During the performance of this work one of us (J.E.) was şupported in part by NSF Grant GP 20033, and the other (W.R.) by US Air Force Grant AF-AFOSR-903-67 and NASA Grant NGL 44-004-001.

\section{References}

[1] H. Thirring, Physik. Z. 19 (1918) 33: correction in Physik, Z, 22 (1921) 29.

[2] L. Bass and F. A. E. Pirani. Phil. Mag. Ser. 7 46 (1955) 850 .

[3] H. Hön1 and A.-W. Maue, Z. Physik 144 (1956) 152.

[4] D. R. Brill and J. M. Cohen. Phys. Rev. 143 (1966) 1011; Nuovo Cim. Ser. X. 56B (1968) 209.

[5] J. M. Cohen, Astrophys, and Space Sci. 6 (1970) 263

[6] W. Rindler, Essential relativity (Van Nostrand Reinhold, 1969) p. 16.

[7] K.-D. Hofmann. Z. Physik 166 (1962) 567

[8] H. Dehnen, Z. Physik 191 (1966) 335.

[9] H. Reissner, Ann. Physik 50 (1916) 106. 